\title{
NEUROTIC TENDENCIES IN EPILEPSY
}

\author{
BY \\ MARGARET DAVIES EYSENCK* \\ From the Neurological Research Unit of the Medical Research Council, \\ National Hospital, Queen Square, London
}

Many psychiatrists believe that there are special personality traits characteristic of the epileptic. Various writers have referred to these as mental peculiarities, emotional instability, impulsiveness, moral anergia, and incapacity for productive occupation involving initiative and consecutive activity (Spratling, 1904), eccentricity, supersensitiveness, emotional poverty, and rigidity (Clark, 1914), lack of intimate friends, solitariness, lack of gregariousness, tactlessness (Clark, 1918), moroseness, indifference, circumstantial and stereotyped manner of speaking, and limited content of speech, egocentricity, irascibility, hypersociability, and religious devotion (Fleck, 1934). Many psychologists hold the view that a special epileptic syndrome exists, and have even, like Humm and Wadsworth in 1934 and Cattell in 1936, incorporated a special epileptic character-scale into their measuring devices. While this belief has not gone unchallenged there seems to be little objective evidence available with which to assess the value of this conception.

If it were found that epileptics differ from normals in various respects the further question arises whether this deviation is due to endogenous causes or is reactive to the disease process itself. The former belief is held by Clark (1918), Clark and Cushing (1931), MacRobert (1916), Jelliffe (1935), and others.

This paper presents an experimental investigation of a hypothesis which may be formally stated thus : "Patients suffering from epileptic seizures tend to be emotionally unstable, immature, and neurotic persons". The hypothesis requires that epileptics shall be differentiated significantly from normal groups on tests known to measure neuroticism.

\section{Experiment}

Population.-Thirty-eight patients, 20 women and 18 men, who attend for the treatment of epilepsy at the National Hospital, London, were tested in groups of not more than 5. Eighteen had grand mal attacks, seven petit mal, and 13 had both types of seizures : so far as could

* Working on behalf of the Medical Research Council. be ascertained all were cases of idiopathic epilepsy. Their average age at the time of the group testing was 23.2 years. Twenty-three of them had left school at the age of 14, and four had stayed on until $17 ; 22$ attended elementary schools, eight central schools, four technical schools, two public schools, one a private school, and one had been at an epileptic colony. On the abstractions test of the Shipley-Hartford scale their average mental age was 13.8 ; on the vocabulary test of the same scale their verbal mental age was 15.9. These figures are within normal limits. Several years before this group testing was carried out, 23 of the patients had been given individual mental tests : at that time their average age was 18.6 ; their average percentile score on the progressive matrices was 24.4 ; and their verbal mental age on the Mill Hill vocabulary test was 17-2. In other words, at that time these 23 patients were, on the average, mentally slightly below the average of a normal group, whose matrices percentile would be 50 and whose verbal mental age would equal their chronological age, i.e. 18.6.

In order to reduce the danger of biased sampling in this study, the cases were selected in such a way that any bias would have tended to go against rather than in favour of the theory, by excluding all patients who were mentally defective, incapable of paid employment, or particularly irregular in their attendance at the clinic.

Tests.-Three specific tests were used.

Word.Connexion List (Crown, $\uparrow 1947)$. - This consists of 50 stimulus words presented together with two alternate responses for each ; e.g., "Sink : wash drown". The subject is asked to underline one of the two responses, whichever is more connected in his mind with the stimulus word. From the choices made by normal and by neurotic groups it has been thought possible to label one response "normal" and the other " abnormal". The score of any subject is the number of abnormal responses underlined.

Maudsley Questionnaire (Eysenck, $\ddagger 1947$ ). - This contains 40 questions, to be answered "yes" or " no", e.g., "Do you have dizzy turns ?" Most of the questions deal with largely physical complaints and a few psycho† I am grateful to Mr. Crown for letting me have more extensive
and more recent figures for normal groups than are available in the and more recent figle referred to.

I am indebted to $H$. J. Eysenck for providing me with the most recent figures obtained on a larger and better selected sample of normal subjects than that reported in his book. 
logical questions are also included. The score is the number of "yes" answers underlined. It has been found that neurotic groups tend to have a larger number of " yes" responses than a stable group.

Ranking Rorschach Test (Eysenck, 1947).-Each patient is given a set of the 10 Rorschach cards and also a list of nine possible responses for each of the ink-blots. $\mathrm{He}$ is required to write a 1 after the response which seems to him most like the ink-blot and also to number the remaining eight in order of resemblance to the inkblot. There are considered to be four neurotic and five normal responses for each of the 10 ink-blots. The summed ranks of the four neurotic responses for each of the 10 cards is taken to be the subject's score. (Absolute normal score-300 ; absolute neurotic score-100.)

All three tests have been shown to differentiate very significantly between normal and neurotic groups, and they should be sufficiently reliable to detect distinct tendencies in the group studied, particularly as the three tests differ considerably in their make-up, in their obviousness, and in the ease with which the tester can be deliberately misled. The Maudsley questionnaire lends itself most easily to falsification, the word connexion list much less so, and the ranking Rorschach test probably least of all, as its underlying purpose is very rarely understood by the subjects.

While the groups on whom the norms were established were normal in the sense that none of the members was actually under psychiatric observation at the time, the figures given by Russell Fraser (1937) have shown that in any such group a proportion of at least $10 \%$ is relatively severely disabled by emotional conflict, a fact which will attenuate any differences that may be found.

In the case of the norms used for the Maudsley questionnaire, the subjects had been seen by a psychiatrist, who divided them into three groups : "good", "doubtful", and "poor". For the comparison the average and standard deviation of the "good" group have been used. Therefore, in this test the attenuation mentioned above will not be present to the same extent as in the other two tests. The average and standard deviation for the total group, including " good", " doubtful ", and " poor" are also shown, so that the reader may have the full details available.

Results.-The results were as follows.

Word Connexion List.-The average score of the epileptic group was $10 \cdot 6$, with a standard deviation of 5.4 compared with the average for the normal group of 7.5, standard deviation 3.9 ; that is, the epileptic subjects gave on the average three more "abnormal" responses than the normal group. The probability of such a difference occurring by chance is less than 1 in $100(\mathrm{P}<0.01)$.
Maudsley Questionnaire.-The average score of the epileptic group was $14 \cdot 2$, with a standard deviation of 7.7 compared with the average for the "good" normal group of $8 \cdot 3$, standard deviation 6.0 ; that is, the epileptic subjects gave on the average nearly six more "yes" responses than the " good" normal group. The probability of such a difference occurring by chance is less than 1 in 100 $(\mathrm{P}<0.01)$.

The difference between the epileptics and the total normal group, however, falls below the level of significance, for with the total normal group, including "good", doubtful", and "poor", the average is 11.6 and the standard deviation 8.6.

Ranking Rorschach Test.-The average score of the epileptic group was 202, with a standard deviation of 33 compared with the average for the normal group of 231, standard deviation 26 . The probability of such a difference occurring by chance is less than 1 in $100(\mathrm{P}<0.01)$. The epileptic average is slightly more indicative of neuroticism than that reported for the neurotic patients at Mill Hill Hospital, which was 205 ; standard deviation 26.

It is apparent, therefore, that all three tests bear out the hypothesis at a high level of statistical confidence, and that in each case the mean of the epileptic group is found to be displaced roughly one standard deviation from the mean of the normal group towards the neurotic end of the scale, the actual values being difference on word connexion list $0.79 \sigma$; difference on Maudsley questionnaire $0.98 \sigma$; difference on ranking Rorschach test $1 \cdot 10 \sigma$. The average difference was $0 \cdot 96 \sigma$. This agreement between the three tests strongly supports the original hypothesis.

The difference on the Maudsley questionnaire using the total group including " good ", " doubtful ", and "poor" is $0.30 \sigma$. The Maudsley questionnaire is the easiest of the three tests to falsify as its purpose is quite obvious. It is noteworthy that the differentiation between the normals and epileptics, when the total group of normals is considered, is least on the questionnaire, showing that the epileptics were trying to make a good impression rather than trying to show the investigator with how many ills they were afflicted. This finding of a direct relationship between discrimination, on the one hand, and lack of obviousness of the measuring instrument, on the other, suggests that the neurotic tendency found is not in any way due to an effort, conscious or unconscious, on the part of the patients to falsify their responses. In this connexion, the intercorrelations of the three tests for the epileptic group may be of interest. They are word connexion list versus Maudsley questionnaire $=0.41$; word connexion list versus ranking Rorschach test $=$ 
0.13 ; Maudsley questionnaire versus ranking Rorschach test $=0.01$.

\section{Discussion}

The results have shown that a group of epileptic men and women, selected in the manner explained above, is differentiated significantly from various normal groups on three tests of neuroticism. The possibility exists that epileptics may have some other mental disorder which would give results differing from normals in the same way as neurotics. These tests have not been given to groups with organic deterioration or to psychopaths, so it is not known whether or not such patients would show similar differences in their results. Therefore, it is not possible to be dogmatic in equating the difference found with neuroticism, but it may be accepted as at least a possible explanation. If it be so accepted, then it may be of interest to see whether the results of this investigation support either of the following theories: (a) that epilepsy is more likely to occur and develop in a person predisposed to neuroticism, or $(b)$ that epilepsy tends to produce neurotic concomitants in a previously normal person.

It was thought that evidence might be contained in the data relating to the duration of the illness compared with the degree of neuroticism. In order to get a single measure of neuroticism, the scores in the three tests were combined by a rough weighting procedure designed to equalize their contributions to the total score by equalizing their standard deviations. When this total score of neuroticism was correlated with the length of time of the illness the correlation was found to be completely insignificant, $(r=0.09)$, showing that length of illness was not in any way correlated with degree of neuroticism. This finding is incompatible with theory $(b)$ and would seem to lend support to the belief that the endogenous rather than the reactive hypothesis is correct. This view is strengthened in some degree by the fact that a slightly higher correlation was found between early onset of the disorder and high neuroticism score $(r=0.25)$. While this correlation is not significant statistically it is suggestive, and if duplicated in further work would seem to give additional support to the hypothesis.

It is interesting, although not strictly relevant to the hypothesis, that the neuroticism score showed a slight negative correlation with the two intelligence tests used, namely, the abstraction and vocabulary parts of the Shipley-Hartford scale. For the abstraction test the correlation was -0.31 , which is significant at the $5 \%$ level, while for the vocabulary test the correlation was $-\mathbf{0 \cdot 2 8}$, which is significant at the $6 \%$ level. These values are very similar to those given for the correlation between intelligence and neuroticism by Eysenck (1937).

A conceptual quotient (C.Q.) was calculated for each patient. C.Q. is the ratio of the patient's corrected abstraction-age to that of a " normal" person receiving his vocabulary score. The answer is multipled by 100 to eliminate decimals. It was found that the C.Q., which is frequently used as a measure of deterioration, was 88 on the average, whereas for a normal group the average would be 100. The C.Q. correlated with the neuroticism score to the extent of $-\mathbf{0 . 2 8}$, which is significant at the $6 \%$ level, and might be taken to indicate a higher degree of deterioration for the more neurotic patients, although in view of the great difficulties associated with the interpretation of such quotients no great reliance should be placed in this finding.

\section{Summary}

The experiment described in this paper was designed to investigate the hypothesis that patients suffering from epileptic seizures tend to be emotionally unstable, immature, and neurotic persons.

Three tests known to differentiate neurotic from normal groups were given to 38 epileptic patients, 20 female and 18 male, whose average age was 23 and who were of roughly average intelligence.

When test results were compared with those of normal groups it was found that on all three tests the average score of the epileptic group was roughly one standard deviation below that of the normal groups. This difference was tentatively equated with neuroticism.

It was shown that there was no correlation between length of illness and degree of neuroticism, a result which would seem to negative the theory that neuroticism in the epileptic is merely a reaction to his illness.

It was also shown that the more neurotic subjects tended to have lower abstract intelligence scores, lower vocabulary scores, and lower conceptual quotients on the Shipley-Hartford scale. (The conceptual quotient is often taken to indicate intellectual deterioration.)

The results of the experiment may thus be interpreted as supporting the original hypothesis, and it is concluded that epileptics tend to have a stronger neurotic predisposition than non-epileptics.

My thanks are due to Dr. E. A. Carmichael for his constant interest and helpfulness, and to Mrs. Whitelaw for her assistance. 


\section{REFERENCES}

Cattell, R., B. (1936). "A Guide to Mental Testing. London." University of London Press.

Clark, L. P. (1914). Amer. J. med. Sci., 148, 729

(1918). J. Amer. med. Ass., 70, 357.

(1918). Amer. J. med. Sci., 155, 796.

- , and Cushing, K. (1931). Med. J. Rec., 133, 27.

Crown, S. (1947). J. Personality, 16, 198.

Eysenck, H. J. (1947). "Dimensions of Personality." London: Kegan Paul, Trench, Trubner.
Fleck, U. (1934). Arch. Psychiat. Nervenkr., 102, 383.

Fraser, R. (1947). "The Incidence of Neurosis among Factory Workers." Industrial Health Research Board. Report No. 90. H.M.S.O. London.

Humm, D. G., and Wadsworth, G. W. (1934). Personnel $J ., 12,314$.

Jelliffe, S. E. (1935). Amer. J. Psychiat., 92, 565.

MacRobert, R. G. (1916). Med. Rec., N. Y., 89, 856.

Spratling, W. P. (1904). " Epilepsy and its Treatment." Saunders, Philadelphia. 